\title{
Reduced IL-4 associated antibody responses to vaccine in early pre-diabetes
}

\author{
S. Schmid ${ }^{1}$, A. Molteni ${ }^{2}$, M. Füchtenbusch ${ }^{1}$, H. E. Naserke ${ }^{1}$, A.-G. Ziegler ${ }^{1}$, E. Bonifacio ${ }^{2}$ \\ ${ }^{1}$ Diabetes Research Institute and $3^{\text {rd }}$ Medical Department, München-Schwabing Hospital, Munich, Germany \\ 2 Department of Medicine, San Raffaele Sientific Institute, Milan, Italy
}

\section{Abstract}

Aims/hypothesis. The aim of this study was to determine whether beta-cell autoimmunity is associated with immune response bias to exogenous antigens.

Methods. IgG subclass responses against tetanus toxoid and rubella were measured after vaccination in children with $(n=36)$ and without $(n=73)$ islet autoantibodies participating in the BABYDIAB prospective study of offspring of parents with Type I (insulindependent) diabetes mellitus. All children had been vaccinated against tetanus toxoid antigen before 6 months of age and at 18 months of age, and against live attenuated rubella virus at 18 months of age and again before 5 years of age. Tetanus toxoid specific IgG subclasses and cytokine responses were compared in a second cohort of subjects.

Results. Responses to tetanus toxoid in islet-autoantibody-negative children were characterized by early IgG1 antibodies at 9 months of age followed by the appearance of $\mathrm{IgG} 4$ and lesser $\mathrm{IgG} 2$ antibodies at
2 years of age. Children who had developed islet autoimmunity before one year of age $(n=15)$ did not have the shift to IgG4 and IgG2 anti-TT after booster vaccination $(p<0.01)$, and had undetectable or IgG1 restricted responses. This defect was independent of HLA class II genotype, was restricted to children who had islet autoimmunity before 1 year of age, and was most evident in children who already had multiple islet autoantibodies by 9 months of age. IgG4 and $\mathrm{IgG} 2$ anti-TT correlated with IL-4 $(p<0.005)$, but not IFN $\gamma$ responses. Antibody responses to the IFN $\gamma$-inducing rubella vaccination were strongly $\mathrm{IgG} 1$ dominated and no differences were observed between islet autoantibody positive and negative children.

Conclusions/interpretation. These data are consistent with a reduced capacity to make IL-4 promoted antibody responses to exogenous antigen in early pre-diabetes. [Diabetologia (2002) 45:677-685]

Keywords Diabetes, autoimmunity, autoantibodies, isotype switching, vaccination.
Received: 30 November 2001 / Revised: 24 January 2002

Published online: 17 April 2002

(C) Springer-Verlag 2002

Corresponding author: E. Bonifacio, Department of Medicine 1, Istituto Scientifico San Raffaele, Via Olgettina 60, I-20132 Milan, Italy, e-mail: bonifacio.ezio@hsr.it

Abbreviations: IFN $\gamma$, Interferon-gamma; DTP, diphtheria tetanus pertussis vaccine; Th, T helper; TT, tetanus toxoid; 9 mo AutoAb+, offspring with persistent islet autoantibodies at 9 months of age; $2 \mathrm{yr}$ AutoAb+, offspring with persistent islet autoantibodies at 2 years of age but not at 9 months of age; AutoAb neg, offspring with no detectable islet autoantibodies at 9 months and 2 years of age; MMR, measles mumps rubella; anti-TT, antibodies to tetanus toxoid
Autoimmune diabetes is a T-cell-mediated disease [1]. In animal models of the disease, autoimmunity is characterised by interferon-gamma (IFN $\gamma$ ) dominated immune responses to beta-cell autoantigens [2, 3, 4], impaired regulatory mechanisms and reduced interleukin-4 (IL-4) responses which could lead to a generalized immune deviation $[5,6]$.

In humans, evidence that Type I (insulin-dependent) diabetes mellitus is associated with a generalized skewing away from IL-4-mediated responses is provided by the observation that patients with Type I diabetes or autoimmunity to beta-cell antigens have a reduced prevalence of allergy $[7,8]$. Another model to investigate generalized immune deviation is represent- 
ed by the immune response to vaccination. Vaccination against both bacterial and viral infectious agents is done early in life on a large scale, representing an early environmental challenge to the immune system. The BABYDIAB study provided an opportunity to study immune responses to vaccine antigens with respect to diabetes risk. Children of parents with Type I diabetes are followed from birth with blood samples at 9 months, 2 years and 5 years of age [9]. Over 1500 children have been enrolled and $2.7 \%$ have developed persistent diabetes-associated islet autoantibodies [10], making it possible to determine whether early beta-cell autoimmunity is associated with a general immune bias. As model antigens, tetanus and rubella were chosen for this study. Children were vaccinated against tetanus together with diphtheria and pertussis (DTP) bacterial antigens before 6 months of age with a subsequent booster at around 18 months of age, and the majority of children were also vaccinated with live attenuated rubella virus between age 1 and 2 years and then again before 5 years of age. Immune responses to the DTP bacterial antigens are expected to be mediated by both IFN $\gamma$ and IL-4, whereas those against rubella are expected to be predominantly mediated by IFN $\gamma$. In the current study, antigen-specific antibody subclasses were used to measure the magnitude and quality of responses to these antigens. The subclass response is an indirect measure of T-cell phenotype in the mouse $[11,12]$, while in humans IgG4 or IgE antibody production or both are promoted by the T helper (Th) 2 cytokines IL-4 and IL-13 [13, 14, 15, 16]. Our finding of a marked impairment in the IL-4 associated IgG4 response to tetanus toxoid (TT) in children with early established pre-diabetes supports the concept of a generalized immune deviation in the presence of beta-cell autoimmunity.

\section{Methods and materials}

Subjects. Serum samples used for measurement of TT antibodies in this study were obtained from the German BABYDIAB study [10]. BABYDIAB is a prospective study from birth in more than 1500 offspring of parents with Type I diabetes and regular blood sampling at birth, 9 months, 2,5 , and 8 years of age scheduled in all offspring. Offspring are tested for autoantibodies to insulin, GAD, the protein tyrosine phosphatase IA-2, and islet cells on all visits. At 2 years of age samples are also collected for HLA typing [17]. Of the 959 children followed until age 2 years, all have completed vaccination against TT, and of the 381 children followed until age 5 years, 285 have completed vaccination against rubella [18]. For this study, all children who had received tetanus or rubella vaccination and had developed persistent non-maternally acquired islet autoantibodies were included (Table 1). Of these, 15 had developed islet autoantibodies already by 9 months of age (9 mo AutoAb+ offspring), and a further 21 were islet autoantibody negative at age 9 months but developed persistent islet autoantibodies at 2 years of age ( $2 \mathrm{yr}$ AutoAb+ offspring). Thirteen of the islet autoantibody positive children have developed Type I diabetes between age 1.3 to 8.8 years. The high risk $H L A \quad D R B 1 * 03 \quad D Q B 1 * 0201 / D R B 1 * 04 \quad D Q B 1 * 0302$ $(D R 3 / 4)$ or HLA DRB1*04 DQB1*0302/DRB1*04 DQB1*0302 $(D R 4 / 4)$ genotypes were present in 16 of the islet autoantibody positive children. In order to control for potential HLA associated differences in the immune response to vaccination, all islet autoantibody-negative offspring with high risk genotypes $(n=42)$ and 31 randomly selected autoantibody negative offspring without these genotypes were included for comparison in this study. None of the islet autoantibody negative children have developed Type I diabetes. DTP vaccination was given at around 3 months, 4 months, 5 months and 18 months of age in all but seven offspring (three autoantibody positive and four autoantibody negative) who received DT only at these time points. Antibodies to tetanus were measured in 9-month and 2-year serum samples. Measles, mumps and rubella (MMR) vaccination was given between 12 and 24 months of age followed by a booster vaccination before 5 years of age in 82 of the children included in this study. Antibodies to rubella antigen were measured in the 5-year samples from these children and from 20 offspring who were not vaccinated with MMR and had not reported rubella infection.

Both T-cell and antibody responses to TT were measured in a separate cohort of 31 subjects in whom samples could be obtained for T-cell studies. These 31 subjects included relatives from the BABYDIAB study and the Munich family study [19] in whom both T-cell and antibody studies could be performed at the same age. All subjects had completed vaccination against DTP. Subjects had a median age of 7.3 years, 24 had islet autoantibodies and 7 developed Type I diabetes.

Anti-vaccine Antibodies. IgG subclass antibodies to TT (antiTT) were measured by an ELISA similar to that used in the World Health Organisation (WHO) evaluation of the WHO standard [20]. TT antigen used for antibody measurement was identical to that used in vaccine preparations. Flat bottom 96-well plates were coated with $0.5 \mathrm{LF} / \mathrm{ml}$ of TT antigen (Chiron Behring, Marburg, Germany) in $125 \mu \mathrm{l}$ of $147 \mathrm{mmol} / \mathrm{l}$ $\mathrm{NaCl}, 1.5 \mathrm{mmol} / \mathrm{l} \mathrm{KCl}, 2.7 \mathrm{mmol} / \mathrm{l} \quad \mathrm{KH}_{2} \mathrm{PO}_{4}, 81 \mathrm{mmol} / \mathrm{l}$ $\mathrm{Na}_{2} \mathrm{HPO}_{4} \mathrm{pH} 7.4$ (PBS) by overnight incubation at $4{ }^{\circ} \mathrm{C}$. Plates were washed three times in PBS containing $0.05 \%$ Tween 20 (PBST) and blocked in $0.1 \%$ gelatin in PBS. Sera were diluted $1 / 200$ (total $\mathrm{IgG}$ ), 1/800 (IgG1), and $1 / 400$ (IgG2, IgG3, and IgG4) in PBST, $100 \mu \mathrm{l}$ added to duplicate antigen coated wells and duplicate uncoated wells, and were incubated for $2 \mathrm{~h}$ at room temperature. Wells were washed two times with PBST and $100 \mu \mathrm{l}$ of specific biotinylated monoclonal antibody to total IgG, IgG1, IgG2, IgG3 or IgG4 (Pharmingen, San Diego, Calif., USA) diluted $1 / 1000$ in PBST were added. Plates were incubated for $2 \mathrm{~h}$ at room temperature, washed two times with PBST and $100 \mu \mathrm{l}$ of peroxidase conjugated streptavidin (Pharmingen) diluted 1/1000 in PBST were added. After 1-h incubation, plates were washed three times with PBST, colour substrate (ABTS, Sigma-Aldrich, St Louis, Miss., USA) added, and the $\mathrm{OD}_{405}$ of each well measured after 15 min incubation at room temperature. The OD of binding by sera to wells without antigen (median $\mathrm{OD}=0.33$, range $0.31-0.35$ ) was subtracted for each sample. IgG1 subclass anti-TT ODs were multiplied by two to allow for the two-fold higher serum dilution used in comparison to the remaining subclass measurements. Serial dilutions of the WHO reference standard were included in each assay to control for inter-assay variation. Dilutions showed linearity over the range of measurement. The interplate CVs for IgG1-, IgG2-, and IgG4-anti-TT were $4.3 \%$, $5.6 \%$, and $8.0 \%$, respectively. Thresholds for IgG subclass anti-TT responses were determined using frequency histogram distributions and q-q plots [10] of all offspring results. For each subclass, the results showed a cohort with a normal distri- 
Table 1. Islet-autoantibody-positive children included in the study

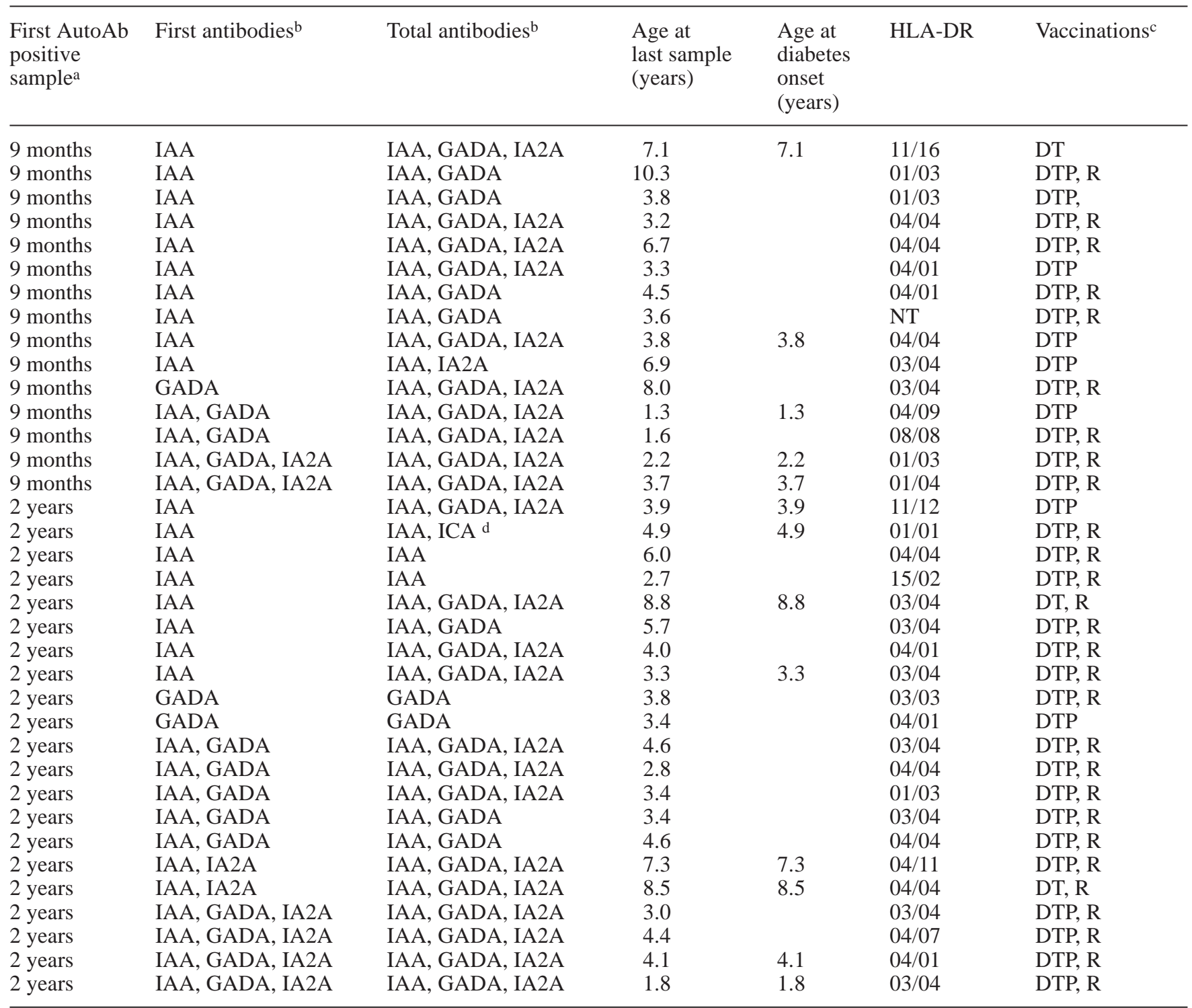

a Children with antibodies at age 9 months were classified as 9 mo AutoAb + offspring; children who were first autoantibody positive at age 2 years were classified as $2 \mathrm{yr}$ AutoAb + offspring b First antibodies indicate diabetes-associated autoantibodies detected in the first autoantibody positive sample; total antibodies indicate autoantibodies detected in all samples tested
${ }^{c}$ DTP indicates vaccination against diphtheria, tetanus and pertussis, $\mathrm{R}$ indicates vaccination against rubella. Other vaccinations are not shown

d ICA is shown only when positive in the absence of GADA and IA2A bution and for IgG1, IgG2, and IgG4 a second cohort with higher results. The upper range of the normal distributions corresponded to ODs of 0.3 for $\operatorname{IgG} 1,0.25$ for $\operatorname{IgG} 2,0.15$ for IgG3, and 0.10 for IgG4, and these were used as the threshold for indicating a specific antibody response. Validating these thresholds, the ODs of 20 sera from subjects who had not been vaccinated or did not have any IgG TT antibodies ranged from -0.03 to 0.27 for $\operatorname{IgG} 1,0.03$ to 0.26 for $\operatorname{IgG} 2,-0.11$ to 0.08 for $\mathrm{IgG} 3$, and -0.02 to 0.08 for $\mathrm{IgG} 4$.

Human IgG subclass antibodies to Rubella-virus were measured by ELISA (Enzygnost Anti-Rubella-Virus/IgG, Dade Behring, Marburg, Germany). Sera were diluted 1/100 (IgG1) or $1 / 50$ in PBST, $100 \mu \mathrm{l}$ were added to duplicate wells coated with antigen (produced from a virus infected cell line) or con- trol antigen (produced from an uninfected cell line), and were incubated for $2 \mathrm{~h}$ at room temperature. Wells were washed three times with PBST and $100 \mu \mathrm{l}$ of specific biotinylated monoclonal antibody against human IgG1, IgG2, IgG3 and IgG4 (Pharmingen) diluted $1 / 500$ in PBST were added. Wells were incubated for $2 \mathrm{~h}$ at room temperature, washed three times with PBST and $100 \mu \mathrm{l}$ of $1 / 1000$ dilution of peroxidase conjugated streptavidin (Pharmingen) added. After 1-h incubation, wells were washed three times with PBST, $100 \mu$ l of colour substrate (TMB Peroxidase Substrate Kit, Bio-Rad, Munich, Germany) added. After $10 \mathrm{~min}$ incubation at room temperature, $100 \mu \mathrm{l}$ of $4 \mathrm{~N} \mathrm{H}_{2} \mathrm{SO}_{4}$ was added to stop the reaction and the $\mathrm{OD}_{405}$ of each well measured. Results were obtained by subtracting OD of control antigen from OD of antigen. 
T cell ELISpot assays. PBMC were obtained by density gradient centrifugation over Ficoll Paque (Pharmacia Biotech, Freiburg, Germany), isolated from the gradient interface, washed twice in RPMI-1640 medium supplemented with 5\% heat inactivated human $\mathrm{AB}$ positive serum, $2 \mathrm{mmol} / \mathrm{l}$ glutamine, $100 \mathrm{U} / \mathrm{ml}$ penicillin, $100 \mu \mathrm{g} / \mathrm{ml}$ streptomycin (Life Technologies, Paisley, UK) and resuspended at $3 \times 10^{6}$ cells $/ \mathrm{ml}$ in the same medium. Cells $\left(3 \times 10^{6}\right)$ were cultured with and without TT $(7.5$ Lf/ml [Statens Serum Institut, Copenhagen, Denmark]) in 48 -well plates for $48 \mathrm{~h}\left(37^{\circ} \mathrm{C}, 5 \% \mathrm{CO}_{2}\right)$. The nonadherent cells were subsequently collected by three gentle washing steps with warm $\left(37^{\circ} \mathrm{C}\right) \mathrm{RPMI}-1640$ medium $+2 \%$ human serum, and resuspended in medium $\left(10^{6}\right.$ cells $\left./ \mathrm{ml}\right)$. For the enumeration of antigen-specific cytokine producing cells, wells of an ELISpot plate (U-CyTech, Utrecht, The Netherlands) were coated overnight at $4^{\circ} \mathrm{C}$ with IFN $\gamma$, IL-4 or IL-13specific monoclonal antibody (diluted $1 / 200$ in PBS), washed four times (PBS $+0.05 \%$ Tween-20) and left in PBS $+1 \%$ BSA for $1 \mathrm{~h}$. $100 \mu \mathrm{l}$ of the cell suspension was added to triplicate wells for each cytokine and incubated for $4 \mathrm{~h}$ at $37^{\circ} \mathrm{C}, 5 \%$ $\mathrm{CO}_{2}$. After incubation, cells were discarded, $200 \mu \mathrm{l}$ of deionized water added to each well, plates left on melting ice for $10 \mathrm{~min}$ and washed 10 times with PBST. Secreted cytokine was revealed after incubation with biotinylated anti-cytokine detector antibody $\left(1 \mathrm{~h}, 37^{\circ} \mathrm{C}, 5 \% \mathrm{CO}_{2}\right)$ and enzyme-labelled anti-biotin-antibody, followed by five washes with PBST, colour development $\left(10-15 \mathrm{~min}, 37^{\circ} \mathrm{C}, 5 \% \mathrm{CO}_{2}\right)$, and washing in distilled water. Spots were counted manually under a microscope and expressed as number of spots $/ 500000$ cells after subtraction of spots obtained in wells containing no antigen. Results for cells incubated in the absence of TT were less than 10 spots for 500000 cells for all cytokines in all samples tested.

Statistical analysis. Comparison of antibody titres groups was carried out using the Mann Whitney U test. Comparison of antibody titres at 9 months compared with 2 years was done using the Wilcoxon test for pairs. Correlation between IgG subclass anti-TT titres and between cytokines in ELISpot assays were done using Spearman's non-parametric correlation coefficient. Comparisons of prevalences of antibody responders between groups was done using Fishers exact test. Analyses were also done in the subgroups having the high-risk $D R 3 / 4$ or $D R 4 / 4$ genotypes. For all statistical methods the Statistical
Package for Social Sciences (SPSS, Chicago, Ill., USA) was used. A $p$ value of less than 0.05 (two-sided) was considered to be statistically significant.

\section{Results}

IgG subclass responses to tetanus vaccination in $B A B Y D I A B$ offspring. IgG subclass responses to TT in the BABYDIAB offspring were measured at both 9 months of age (after initial vaccination) and 2 years of age (after booster vaccination). At 9 months of age, $54 \%$ of all the children had anti-TT antibodies above thresholds for positivity (Fig. 1A). Anti-TT antibody prevalence increased to $74 \%$ of all children at 2 years of age after booster vaccination. The predominant IgG subclass response at 9 months of age was IgG1. Low titres of IgG2 or IgG4 anti-TT or both were also found in a minority of offspring. At 2 years of age, IgG1 anti-TT antibodies remained at similar levels, IgG2 antiTT titres increased slightly, whereas IgG4 anti-TT titres increased ( $p<0.001$; Wilcoxon paired test). IgG1 anti-TT were found in $49(47 \%)$, IgG2 anti-TT in 34 (33\%) and IgG4 anti-TT in 68 (65\%) of 104 offspring at 2 years of age. IgG3 anti-TT antibodies were not detected either at 9 months or 2 years of age. No dif-

Fig. 1A, B. A Anti-TT subclass antibody levels in BABYDIAB offspring at 9 months and 2 years of age after vaccination with $\mathrm{DT}(\mathrm{P})$ at $3,4,5$, and 18 mo of age. B Correlation between anti-TT subclass responses in 2 years samples from the BABYDIAB offspring. IgG2 and IgG4 anti-TT responses were highly correlated $(r=0.8, p<0.0001)$, there was only a moderate correlation between IG1 and IgG2 anti-TT $(r=0.46)$ and between IgG1 and IgG4 anti-TT $(r=0.42)$. Horizontal broken lines represent upper limit of normal range for each subclass antibody

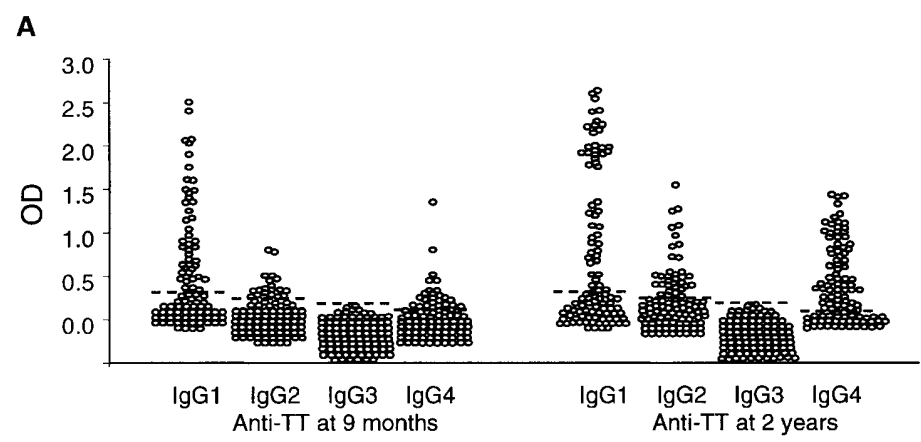

B
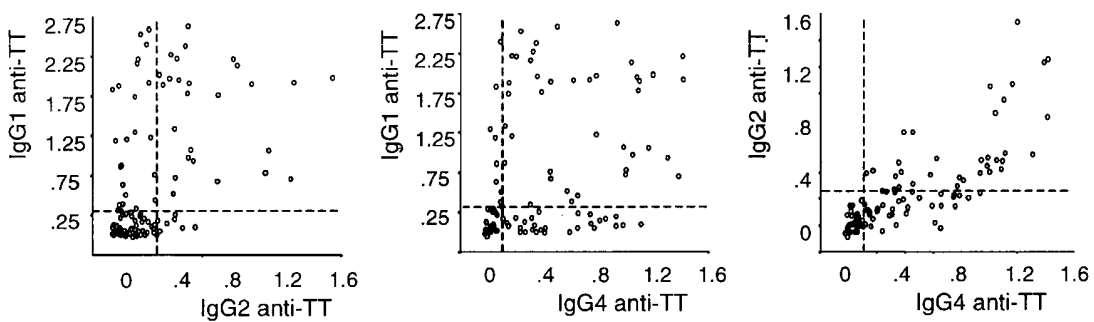
A
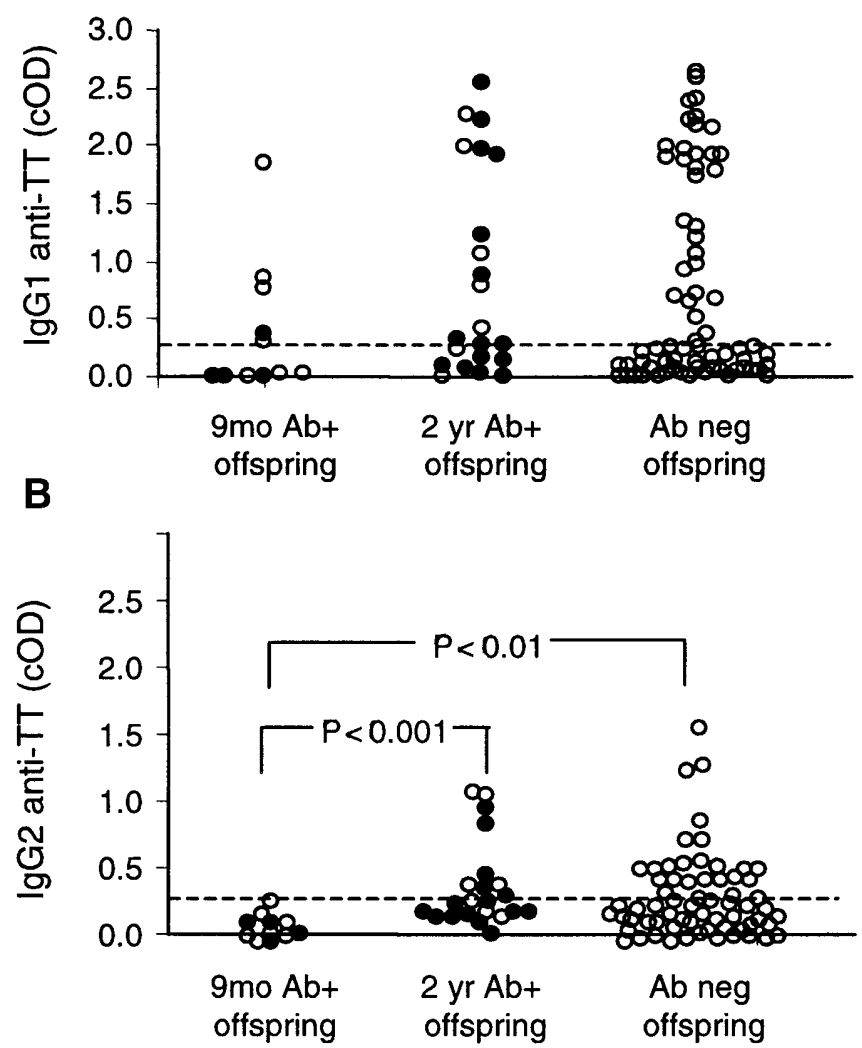

C

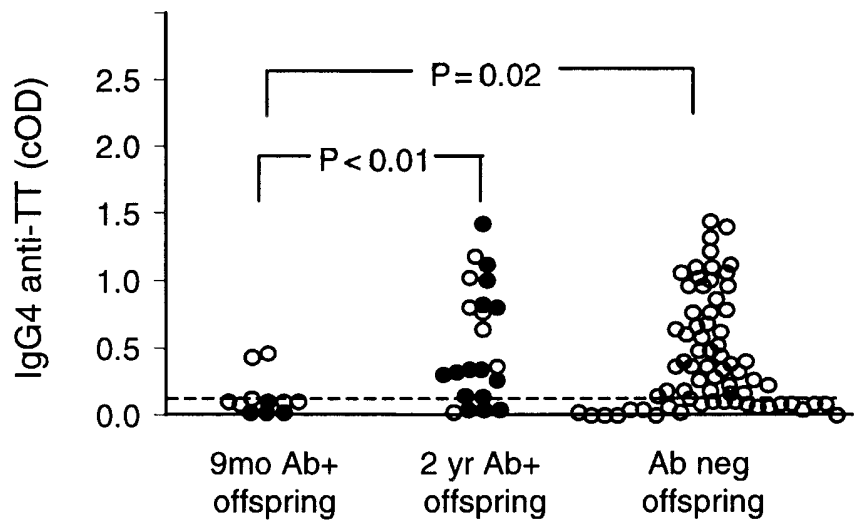

Fig. 2A-C. IgG1 (A), IgG2 (B), and IgG4 anti-TT (C) antibodies in 2 years samples in 9 mo AutoAb+offspring $(n=11)$ compared to $2 \mathrm{yr}$ AutoAb+ offspring $(n=22)$ and AutoAb neg offspring $(n=70)$. Offspring with islet autoantibodies against more than one autoantigen (insulin, GAD, or IA-2). IgG2 and IgG4 anti-TT titres were significantly decreased in 9 mo Auto$\mathrm{Ab}+$ offspring

ferences in anti-TT subclasses were observed with respect to sex of the offspring or proband or HLA class II genotype of the offspring (data not shown).

$\mathrm{IgG} 2$ and $\mathrm{IgG} 4$ anti-TT titres were strongly correlated with each other $(r=0.8, p<0.0001$; Fig. 1B), and all offspring with IgG2 anti-TT had also IgG4 anti-TT. IgG1 anti-TT titres were moderately correlated with IgG2 anti-TT titres $(r=0.46, p<0.001)$ and with IgG4 anti-TT titres $(r=0.42, p<0.001)$.
Anti-TT IgG subclass responses and islet autoimmunity. Both islet autoantibody positive and negative children had predominantly IgG1 anti-TT responses at 9 months of age (data not shown). However, at 2 years of age (after booster vaccination), significantly lower anti-TT responses were found in those offspring who were islet autoantibody positive already at age 9 months (Fig. 2). In particular, the IgG4-anti-TT response did not rise in the 9 mo AutoAb+ offspring (median cOD 0.07; interquartile range $0-0.1$ ) remaining at titres markedly lower than in the $2 \mathrm{yr}$ AutoAb+ offspring (median cOD 0.34; interquartile range $0.12-0.85 ; p<0.01)$ and the AutoAb neg offspring (median cOD 0.31; interquartile range 0.06-0.74; $p=0.02$ ). Moreover, no IgG2-anti-TT response was detected in the 9 mo AutoAb+ offspring $(p<0.001$ vs 2 yr AutoAb+ and $p<0.01$ vs AutoAb neg), and IgG1 anti-TT were also low. Overall, IgG4 or IgG2 anti-TT or both above the threshold for positivity were found in only $2(18 \%)$ of 119 mo AutoAb+ offspring compared to $18(82 \%)$ of $222 \mathrm{yr}$ AutoAb+ offspring $(p<0.001)$ and $50(71 \%)$ of 70 AutoAb neg offspring $(p<0.01)$. The reduced anti-TT responses were most obvious in the 4 offspring who had multiple islet autoantibodies already at 9 months of age. None of these had IgG2 or IgG4 anti-TT and only one had weak IgG1-anti-TT.

The reduced responses in the 9 mo AutoAb+ offspring were not due to $H L A$-associated differences in the anti-TT response: reduced $\operatorname{IgG} 2(p<0.01)$ and IgG4 $(p<0.05)$ anti-TT antibodies in the 9 mo Auto$\mathrm{Ab}+$ offspring compared to the $2 \mathrm{yr}$ AutoAb+ and AutoAb neg offspring were also observed if only children with HLA DR3/4 or DR4/4 genotypes were analysed (data not shown).

Antibody and T-cell responses to TT. T cells were not available in the majority of the BABYDIAB children. Therefore, in order to determine whether there was a relationship between TT antibody subclass responses and $\mathrm{T}$ cell cytokine phenotype, we correlated the cytokine profile of $\mathrm{T}$ cells after stimulation with TT and the IgG subclass of anti-TT antibodies in a separate cohort of 31 subjects (median age 7.3 years; 24 with islet autoantibodies). Utilizing ELISpot measurements, IL-4 and IL-13 responses to TT were strongly correlated ( $r=0.8, p<0.0001$; Spearman's test; Fig. 3). Both IL-4 and IL-13 responses were moderately correlated with IFN $\gamma$ responses $(r=0.55, p<0.01 ; r=0.56$, $p<0.01$, respectively). Ten subjects had relatively high IL-4 and IL-13 ELISpot responses to TT (all but one having also IFN $\gamma$ responses), another 9 had IFN $\gamma$ responses only and 12 had low or absent IFN $\gamma$, IL-4 and IL-13 responses to TT.

IgG4 anti-TT levels correlated with IL-4 responses to TT $(r=0.55 ; p<0.002)$ and IL-13 responses $(r=0.52$; $p<0.005)$, but not IFN $\gamma$ responses $(r=0.1)$. IgG2 antiTT were similarly correlated with IL-4 ( $r=0.61$; 
A

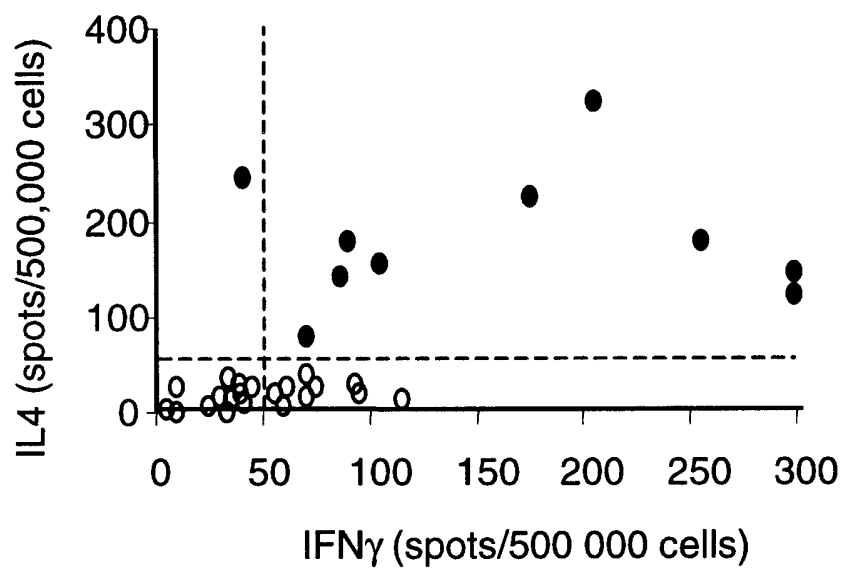

B
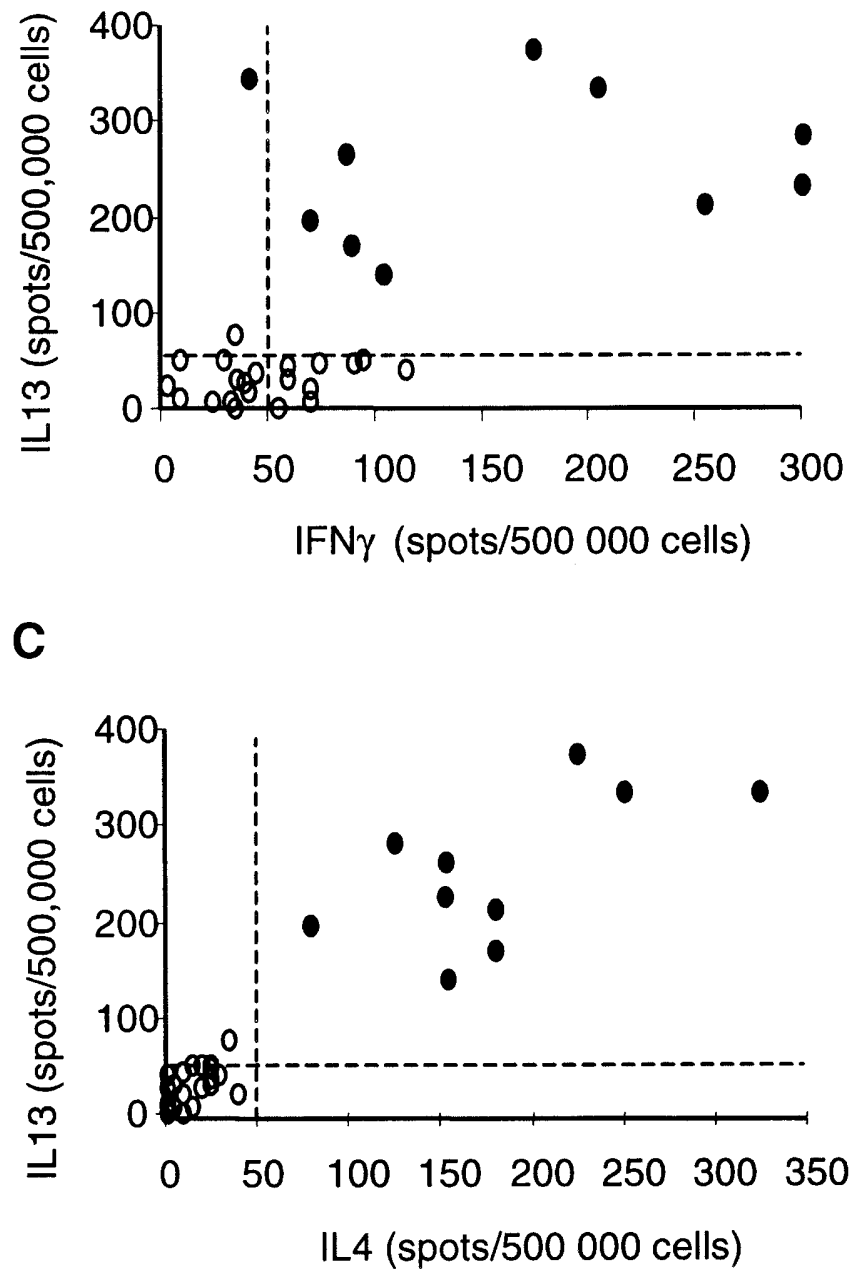

Fig. 3A-C. IFN $\gamma$, IL-4 and IL-13 secretion by PBMCs after stimulation with TT and measured by ELISpot. The broken lines indicate 50 spots for 500000 cells which is the limit of detection of the assay. All subjects had less than 10 spots for 500000 cells in control wells without TT. Subjects who had significant IL-4 and IL-13 responses
A

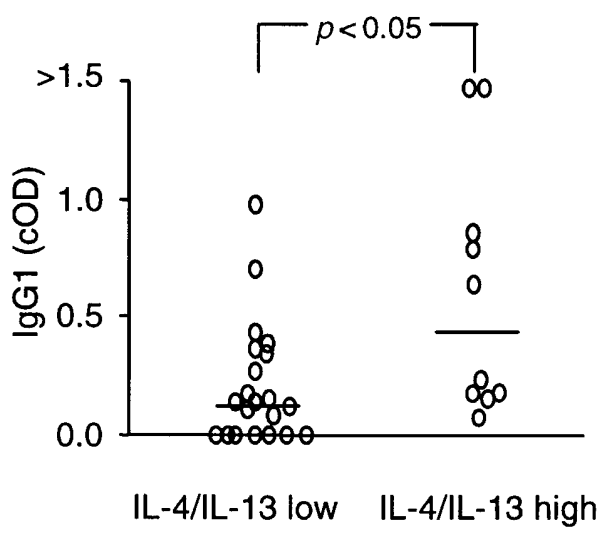

B

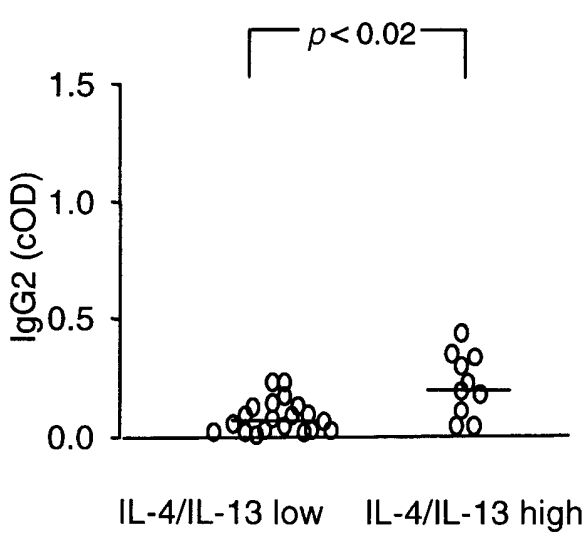

C

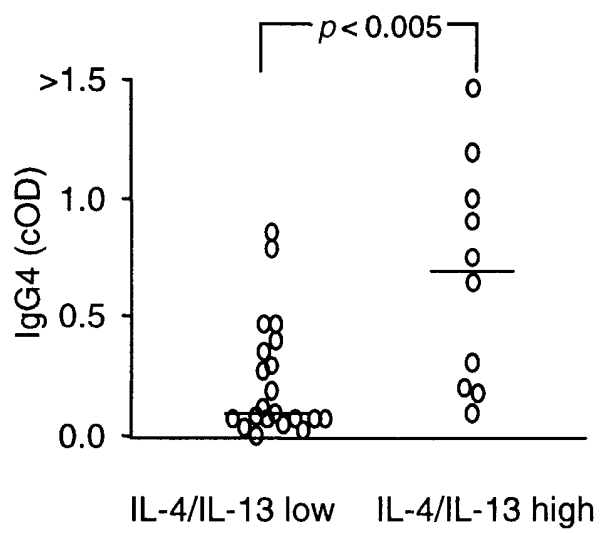

Fig. 4. $\operatorname{IgG} 1$ (A), IgG2 (B) and IgG4 anti-TT (C) titres in subjects with $(n=10)$ and without $(n=21)$ significant IL-4 and IL-13 PBMC responses to TT. All, and in particular IgG4 antiTT $(p<0.01)$ were reduced in subjects without IL-4 and IL-13 responses

$p<0.001)$ and IL-13 $(r=0.47 ; p<0.01)$ and not IFN $\gamma$ $(r=0.1)$, whereas IgG1 anti-TT were not correlated with cytokine responses (data not shown). Accordingly, IgG2 and IgG4 anti-TT antibodies were higher in subjects with high IL-4 and IL-13 ELISpot TT responses (Fig. 4$)$ than those with IFN $\gamma$ only $(p<0.05$ for 


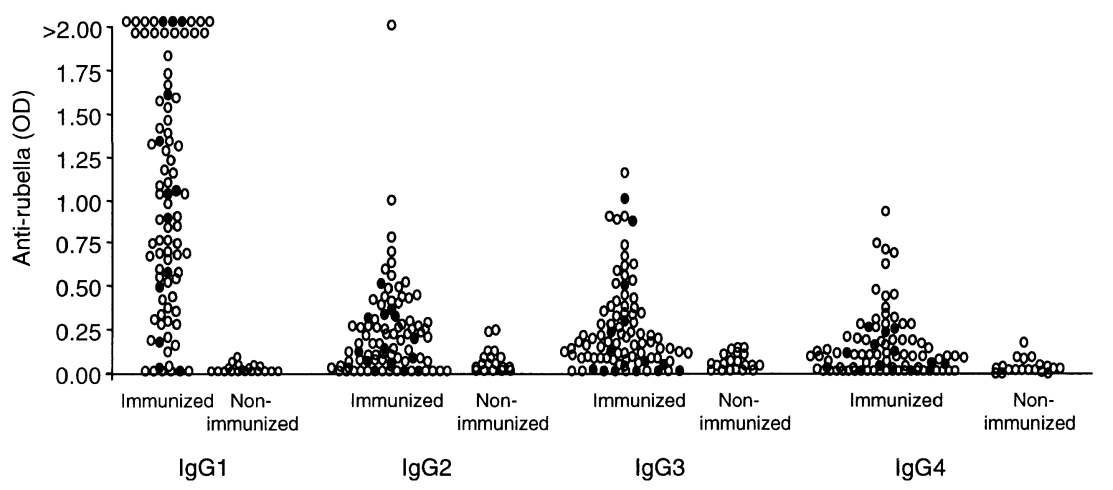

Fig. 5. IgG subclasses of anti-rubella antibodies at 5 years of age in children from the BABYDIAB study who were immunised with live attenuated rubella virus and as control nonimmunised 5-year-old children. Islet-autoantibody positive children are indicated by

$\operatorname{IgG} 2$, and $p<0.01$ for $\mathrm{IgG} 4)$ and those with low or absent ELISpot responses $(p<0.05$ and $p<0.02)$. All 10 high IL-4 and IL-13 TT subjects had detectable anti-TT antibodies (vs 11 of 21 IL-4 and IL-13 low subjects, $p<0.01$ ), all but one had IgG4 anti-TT antibodies (vs 10 of 21 low IL-4 subjects, $p<0.05$ ), and IgG2 antiTT were only found in the high IL-4 and IL13 TT subjects ( $p<0.01$ vs IL-4 and IL-13 low subjects). These data are consistent with an association of $\mathrm{IgG} 4$ and IgG2 antibody responses to TT with the presence of IL-4 and IL-13 secreting TT-specific T cells.

Antibody responses to Rubella vaccine antigen. Rubella vaccine is a live attenuated virus, given in order to promote Th1 cytotoxic immunity to infection. Immunisation is given before 2 years of age and again before 5 years of age. We therefore examined antibody responses in 5-year samples from the BABYDIAB offspring in order to determine whether the $\mathrm{IgG}$ subclass response of a predicted Th1 immunity differed to that against TT. Antibody responses to rubella were strongly IgG1 dominant (Fig. 5). Almost all immunised children had raised IgG1 antibodies to rubella antigen and only a minority also had $\mathrm{IgG} 2, \mathrm{IgG} 3$, or IgG4 antibodies. Unlike the response to TT, IgG3 anti-rubella antibodies above levels found in nonimmunised children could be detected and IgG4 antirubella antibodies were only marginally higher than in non-immunised children. No differences in antibody responses to rubella were found between islet autoantibody positive and negative children and no relationship between responses to rubella and TT were found for individual children (data not shown).

\section{Discussion}

This study investigated the responses to vaccine antigens in young children with increased genetic risk for

Type I diabetes in relation to autoantibody development. It is unique in that vaccination and appearance of islet autoimmunity can be related to each other, something which cannot be done in cross-sectional comparisons of patient and control populations of older subjects. The study shows that antibody responses to TT are generally characterized by early IgG1 antibodies and the appearance of IgG4 and lesser IgG2 antibodies upon further immunisation. Children without islet autoantibodies characteristically had this type of response regardless of their HLA genotype, and this was similar to previous reports for TT [21, 22, 23], and protein antigens such as ovalbumin [24] and keyhole limpet haemocyanin [25, 26]. Children who had islet autoimmunity by one year of age did not have the shift to IgG4 and IgG2 anti-TT after booster vaccination, and consequently had predominantly undetectable or IgG1 restricted responses. This defect was independent of HLA class II genotype. In contrast, children who did not have islet autoantibodies at 9 months of age, but who developed them subsequently (and were therefore autoantibody negative at the time of vaccination), had relatively normal responses to TT which almost always included $\mathrm{IgG} 4$. The observation that reduced $\mathrm{IgG} 2$ and $\mathrm{IgG} 4$ responses to TT were found only in offspring who potentially had ongoing islet autoimmunity at the time of vaccination indicates that such a defect is either acquired, possibly a consequence of the islet autoimmunity, or inherently present in subjects who develop very early islet autoimmunity.

A reduced antibody response to vaccine bacterial antigens suggests either immune suppression or deviation. The reduction was most marked for $\mathrm{IgG} 2$ and $\mathrm{IgG} 4$ and seen only after booster vaccination, implying either a defect in the switching to, or production of, IgG2 and IgG4 upon immunisation with TT. IgG4 production is strongly promoted by IL- 4 and IL-13 [27, 28], and IL-10 further enhances IL-4 induced IgG4 production [29]. The strong correlation we observed between IgG2 and IgG4 anti-TT responses indicates that their production can be influenced by similar factors. Absent or low titres of both $\operatorname{IgG} 2$ and IgG4 are found in the antigen specific response of the IFN $\gamma$-dominated Lyme borreliosis disease [30]. IgG1 restricted responses are also seen in the IFN $\gamma$-dominant response to tuberculosis, whereas IgG2 antibod- 
ies are found in the IL-4-dominant response to leprosy [31]. All these support the concept that IgG4 immune responses are promoted by IL-4. Here, we also examined responses to rubella after vaccination with live attenuated virus in the BABYDIAB offspring. The response to this IFN $\gamma$ inducing vaccine was relatively absent of IgG2 and IgG4 and heavily IgG1 dominated, further suggesting that the IgG4 response is not influenced by IFN $\gamma$. No differences were found in the subclass response to rubella in offspring with islet autoimmunity regardless of when autoantibodies appeared. This observation is consistent with a specific defect in IL-4 associated antibody responses to vaccine antigens in early pre-diabetes.

In order to relate the reduced antibody response to potential cytokine insufficiency at the T-cell level, we examined IgG subclasses of anti-TT and cytokine profiles of T-cell responses to TT. The T-cell responses included high IL-4 and IL-13 in some individuals, and remarkably, these individuals had much higher responses of $\mathrm{IgG} 1, \mathrm{IgG} 2$ and in particular IgG4 anti-TT, a finding consistent with a favouring of antibody responses in Th2 immunity, and with the reported IL-4 dependency of IgG4 production [13, 14, 15, 16].

In conclusion, we have shown an impairment in the ability to produce the IL- 4 related IgG4 antibody response to TT after booster vaccination in individuals who develop islet autoimmunity before 1 year of age. We found IgG4 responses to be a characteristic of antibodies to TT, but virtually absent after vaccination with live attenuated rubella virus, suggesting a Th2 cytokine dependency. Moreover, IgG4 responses were strongly associated with T-cell IL-4 and IL-13 production following stimulation with TT. We suggest that such a defect could be overcome with sustained exposure to antigen because patients with Type I diabetes can readily produce IgG4 antibodies to exogenous insulin [32]. Finally, an IL-4 dependent immune bias would be consistent with recent studies showing reduced number and function of IL-4 secreting NK $\mathrm{T}$ cells very early in NOD mice and humans with Type I diabetes [4, 5, 33].

Acknowledgements. The authors are grateful to A. Knopff for expert technical assistance and to B. Roep for his help in establishing ELISpot assays. This work was supported by grants from the Alexander von Humboldt-Stiftung, the German Research Association (Deutsche Forschungsgemeinschaft) (ZI310/12-1), the Juvenile Diabetes Research Foundation (1-2000-619), the German Diabetes Society (Deutsche Diabetes-Gesellschaft), the foundation "Das Zuckerkranke Kind", and Telethon (E.587). This study forms part of the dissertation of S. Schmid.

\section{References}

1. Martin S, Wolf-Eichbaum D, Duinkerken G et al. (2001) Development of type 1 diabetes despite severe hereditary B-lymphocyte deficiency. N Engl J Med 345: 1036-1040
2. Katz JD, Benoist C, Mathis D (1995) T helper cell subsets in insulin-dependent diabetes. Science 268: 1185-1188

3. Liblau RS, Singer SM, McDevitt HO (1995) Th1 and Th2 CD4+ T cells in the pathogenesis of organ-specific autoimmune diseases. Immunol Today 16: 34-38

4. Pilstrom B, Bjork L, Bohme J (1995) Demonstration of a Th1 cytokine profile in the late phase of NOD insulitis. Cytokine 7: 806-814

5. Gombert JM, Herbelin A, Tancrede-Bohin E et al. (1996) Early defect of immunoregulatory $\mathrm{T}$ cells in autoimmune diabetes. C R Acad Sci III 319: 125-129

6. Cameron MJ, Meagher C, Delovitch TL (1998) Failure in immune regulation begets IDDM in NOD mice. Diabetes Metab Rev 14: 177-185

7. Douek IF, Leech NJ, Gillmor HA, Bingley PJ, Gale EA (1999) Children with type-1 diabetes and their unaffected siblings have fewer symptoms of asthma. Lancet 353: 1850

8. Olesen AB, Juul S, Birkebaek N, Thestrup-Pedersen K (2001) Association between atopic dermatitis and insulindependent diabetes mellitus: a case-control study. Lancet 357: 1749-1752

9. Roll U, Christie MR, Fuchtenbusch M, Payton MA, Hawkes CJ, Ziegler AG (1996) Perinatal autoimmunity in offspring of diabetic parents. The German Multicenter BABY-DIAB study: detection of humoral immune responses to islet antigens in early childhood. Diabetes 45: 967-973

10. Ziegler AG, Hummel M, Schenker M, Bonifacio E (1999) Autoantibody appearance and risk for development of childhood diabetes in offspring of parents with type 1 diabetes: the 2-year analysis of the German BABYDIAB Study. Diabetes 48: 460-481

11. Snapper CM, Paul WE (1987) Interferon-gamma and B cell stimulatory factor-1 reciprocally regulate $\mathrm{Ig}$ isotype production. Science 236: 944-947

12. Snapper CM, Mond JJ (1995) Towards a comprehensive view of immunoglobulin class switching. Immunol Today 14: $15-17$

13. Lundgren M, Persson U, Lansson P et al. (1989) Interleukin 4 induces synthesis of $\mathrm{IgE}$ and $\mathrm{IgG} 4$ in human B cells. Eur J Immunol 19: 1311-1315

14. Ishizaka A, Sakiyama Y, Nakanishi M et al. (1990) The inductive effect of interleukin-4 on IgG4 and IgE synthesis in human peripheral blood lymphocytes. Clin Exp Immunol 79: 392-396

15. Nusslein HG, Winter M, Trag T, Kalden JR (1991) Interleukin-4 induced IgG subclass and IgE secretion by mononuclear cells from atopic donors. Int Arch Allergy Appl Immunol 95: 257-260

16. King CL, Nutman TB (1993) IgE and IgG subclass regulation by IL-4 and IFN-gamma in human helminth infections. Assessment by B cell precursor frequencies. J Immunol 151: 458-465

17. Schenker M, Hummel M, Ferber K et al. (1999) Early expression and high prevalence of islet autoantibodies for DR3/4 heterozygous and DR4/4 homozygous offspring of parents with Type I diabetes: the German BABYDIAB study. Diabetologia 42: 671-677

18. Hummel M, Füchtenbusch M, Schenker M, Ziegler AG (2000) No major association of breast-feeding, vaccinations, and childhood viral diseases with early islet autoimmunity in the German BABYDIAB Study. Diabetes Care 23: 969-974

19. Dittler J, Seidel D, Schenker M, Ziegler AG (1998) GADIA2-combi determination as first-line screening for improved prediction of type 1 diabetes in relatives. Diabetes 47: 592-597 
20. Sesardic D, Wong MY, Gaines Das RE, Corbel MJ (1993) COLLABORATIVE STUDY, The First International Standard for Antitetanus Immunoglobulin, Human; Pharmaceutical Evaluation and International Collaborative Study. Biologicals 21: 67-75

21. Soo Kim J, Jun Kim S, Jin Shin K, Han Hwang P, Chul Cho S (1989) Changes of tetanus specific IgG, IgM and IgG subclasses after DPT vaccination. Yonsei Med J 30: $158-163$

22. Ruths S, Driedijk PC, Weening RS, Out TA (1991) ELISA procedures for the measurement of $\mathrm{IgG}$ subclass antibodies to bacterial antigens. J Immunol Methods 140: 67-78

23. Devey ME, Bleasdale KM, French MA, Harrison G (1985) The IgG4 subclass is associated with a low affinity antibody response to tetanus toxoid in man. Immunology 55: 565-567

24. Lim PL, Lua YL (1994) Occurrence of IgG subclass antibodies to ovalbumin, avidin, and pneumococcal polysaccharide in children. Int Arch Allergy Immunol 104: 137-143

25. Bird P, Calvert JE, Amlot PL (1990) Distinctive development of IgG4 subclass antibodies in the primary and secondary response to keyhole limpet haemocyanin in man. Immunology 69: 355-360

26. Falconer AE, Friedmann PS, Bird P, Calvert JE (1992) Abnormal immunoglobulin $\mathrm{G}$ subclass production in response to keyhole limpet haemocyanin in atopic patients. Clin Exp Immunol 89: 495-499
27. Kitani A, Strober W (1993) Regulation of C gamma subclass germ-line transcripts in human peripheral blood B cells. J Immunol 151: 3478-3488

28. Punnonen J, de Vries JE (1994) IL-13 induced proliferation, Ig isotype switching, and Ig synthesis by immature human fetal B cells. J Immunol 152: 1094-1102

29. Jeannin P, Lecoanet S, Delneste Y, Gauchat JF, Bonnefoy JY (1998) IgE versus IgG4 production can be differentially regulated by IL-10. J Immunol 160: 3555-3561

30. Widhe M, Ekerfelt C, Forsberg P, Bergstrom S, Ernerudt J (1998) IgG subclasses in Lyme borreliosis: a study of specific IgG subclass distribution in an interferon-gammapredominated disease. Scand J Immunol 47: 575-581

31. Sousa AO, Henry S, Maroja FM et al. (1998) IgG subclass distribution of antibody responses to protein and polysaccharide mycobacterial antigens in leprosy and tuberculosis patients. Clin Exp Immunol 111: 48-55

32. Füchtenbusch M, Kredel K, Bonifacio E, Schnell O, Ziegler AG (2000) Exposure to exogenous insulin promotes IgG1 and the T-helper 2-associated IgG4 responses to insulin but not to other islet autoantigens. Diabetes 49: 918-925

33. Wilson SB, Kent SC, Patton KT et al. (1998) Extreme Th1 bias of invariant Valpha24JalphaQ T cells in type 1 diabetes. Nature 391: 177-181 